\title{
Viscous effects in two-layer, unidirectional hydraulic flow
}

\author{
MARTIN S. SINGH $\dagger$ AND ANDREW McC. HOGG \\ Research School of Earth Sciences, Australian National University, Canberra, ACT 0200, Australia
}

(Received 11 August 2008; revised 22 September 2009; accepted 23 September 2009)

Hydraulic equations are derived for a stratified (two-layer) flow in which the horizontal velocity varies continuously in the vertical. Viscosity is included in the governing equations, and the effect of friction in hydraulically controlled flows is examined. The analysis yields Froude numbers which depend upon the integrated inverse square of velocity but reduce to the original layered Froude numbers when velocity is constant with depth. The Froude numbers reveal a critical condition for hydraulic control, which equates to the arrest of internal gravity waves.

Solutions are presented for the case of unidirectional flow through a lateral constriction, both with and without bottom drag. In the free-slip lower boundary case, viscosity transports momentum from the faster to the slower layer, thereby shifting the control point downstream and reducing the flux through the constriction. However, while the velocity shear at the interface between the two layers is reduced, the top-to-bottom velocity difference of the controlled solution is increased for larger values of viscosity. This counter-intuitive result is due to the restrictions placed on the flow at the hydraulic control point. When bottom drag is included in the model, the total flux may increase, in some cases exceeding that of the inviscid solution.

\section{Introduction}

In the description of high-Reynolds-number flow through a lateral constriction or over topography, a simple treatment is that of internal hydraulics. Traditionally, hydraulic theory is formulated under the assumptions that the flow is steady, hydrostatic and inviscid. The fluid is then considered to consist of a finite number of layers with discrete densities $\rho_{i}$ and velocities $u_{i}$ (see e.g. Armi 1986; Dalziel 1991). The advantage of this approach is that the governing equations can be greatly simplified and can be fully described in terms of the layer Froude numbers $F_{i}^{2}=u_{i}^{2} /\left(g^{\prime} h_{i}\right)$; here $h_{i}$ is the layer thickness and $g^{\prime}$ the reduced gravity. Armi (1986) has given a detailed account of the use of the Froude numbers in analysing inviscid two-layer flows through constrictions and over sills.

An important subclass of solutions to problems of internal hydraulics are flows which are 'hydraulically controlled'. This occurs when the flow organizes itself into a regime in which the value of the Froude numbers are constrained at one or more discrete 'control points'. Physically these points correspond to locations at which the speed of long waves vanishes (e.g. Dalziel 1992), and hence no information can

$\dagger$ Present Address: Program in Atmospheres, Oceans and Climate, Massachusetts Institute of Technology, 77 Massachusetts Avenue, Cambridge, MA 02139-4307, USA

$\ddagger$ Email address for correspondence: Andy.Hogg@anu.edu.au 
propagate upstream through a control. For two-layer flow, the relevant constraint is that the composite Froude number $G$, defined as

$$
G^{2}=F_{1}^{2}+F_{2}^{2}-(1-r) F_{1}^{2} F_{2}^{2},
$$

is unity (Armi 1986). Here, $r$ is the ratio of densities between the upper and lower layers.

An important property of controlled flow is that knowledge of only a few quantities at the control point can give information about the global properties of the flow (Wood 1968). Armi \& Farmer (1986) showed that using inviscid hydraulic theory, a prediction of the maximum flux in a two-layer exchange flow can be calculated with only a few parameters relating to the flow configuration - the exact shape of the constriction or sill is immaterial. However, when viscosity is incorporated into the solution, the flux is reduced from the inviscid prediction, and the reduction depends on the aspect ratio of the channel (Zaremba, Lawrence \& Pieters 2003). For this reason there has been some interest in the problem of incorporating viscosity and mixing into the theory of internal hydraulics (see, for instance, Hogg, Ivey \& Winters 2001a; Gu \& Lawrence 2005). Two different methods have been proposed to accomplish this. The first, which we will refer to as the 'drag model', is to retain the layered formulation of hydraulic theory and parameterize viscous energy dissipation by the velocity difference between the layers (see e.g. Zaremba et al. 2003). The second approach, employed by Garrett (2004), is to opt for a continuous velocity profile and parameterize viscous flux of momentum as a Fickian process.

This paper is concerned with the application of the second method to two-layer flows. In $\S 2$ the method of Garrett (2004) is extended to viscous two-layer flow with a continuous velocity profile, and the numerical technique proposed by Hogg \& Hughes (2006) is applied to this problem. The limitation of this technique is that it depends on the velocity being unidirectional. Nonetheless, we find numerical solutions for the unidirectional case $(\S 3)$, which provides insight into the behaviour of hydraulically controlled flows at finite viscosity.

\section{Theory}

\subsection{Governing equations}

Consider two immiscible layers of fluid with densities $\rho_{1}$ and $\rho_{2}$ flowing through a channel of variable width $b(x)$ and variable bottom height $H(x)$. Subscripts 1 and 2 refer to the lower and upper layers respectively. If the flow is steady and hydrostatic, and cross-channel velocities are negligible, we can write the momentum equation in the $x$-direction for the two layers as

$$
\begin{aligned}
& u u_{x}+w u_{z}=-g\left(H_{x}+h_{1 x}+r h_{2 x}\right)+v u_{z z}, \quad H<z<z_{I}, \\
& u u_{x}+w u_{z}=-g\left(H_{x}+h_{1 x}+h_{2 x}\right)+v u_{z z}, \quad z_{I}<z<z_{F} .
\end{aligned}
$$

Here $\boldsymbol{u}=(u, w)$ is the velocity field; $h_{i}$ is the thickness of layer $i ; v$ is the vertical viscosity coefficient; $g$ is the acceleration due to gravity; and $r$ is the density ratio $\rho_{2} / \rho_{1}$. The layers are numbered from the bottom, and the interface and free-surface heights have been denoted $z_{I}$ and $z_{F}$ respectively. Similarly, we can express mass conservation by

$$
(b u)_{x}+(b w)_{z}=0 .
$$

Following Hogg \& Hughes (2006) we transform the momentum equations into streamline coordinates in order to eliminate the vertical velocity and derive hydraulic 
equations for the system. Defining the coordinates $(X, \eta)$, where $X=x$ and $\eta$ is the height of a streamline at the upstream (left-hand) end of the channel, we can rewrite $(2.3)$ as

$$
\left(b u z_{\eta}\right)_{X}=0
$$

where $w \equiv u z_{X}$ (see Hogg \& Hughes 2006). This equation can be integrated to yield

$$
Q(\eta)=b u z_{\eta},
$$

where $Q$ is defined to be the flux per unit height along a streamline. Similarly, (2.1) and (2.2) become

$$
\begin{array}{ll}
u u_{X}=-g\left(H+h_{1}+r h_{2}\right)_{X}+\frac{v}{z_{\eta}}\left(\frac{u_{\eta}}{z_{\eta}}\right)_{\eta}, & 0<\eta<\eta_{I}, \\
u u_{X}=-g\left(H+h_{1}+h_{2}\right)_{X}+\frac{v}{z_{\eta}}\left(\frac{u_{\eta}}{z_{\eta}}\right)_{\eta}, & \eta_{I}<\eta<\eta_{F},
\end{array}
$$

where $\eta_{I}$ and $\eta_{F}$ are, respectively, the heights of the interface and free surface at the upstream end.

We have assumed in the above derivation that the viscosity coefficient is everywhere constant. This assumption may be violated in cases in which the viscosity is not molecular but is representing, for example, a turbulent eddy transport of momentum. Nonetheless, there is no reason why the solutions derived here could not be easily extended to cases with variable viscosity and/or an embedded turbulent closure scheme. As in Hogg \& Hughes (2006) the horizontal viscosity has been neglected. Thus, the results are only strictly valid for cases in which the channel is wide relative to its depth, and velocities are slowly varying in the streamwise direction. Relaxing these assumptions does not fundamentally change the physics of the situation hydraulic-like equations can still be defined even if terms proportional to $u_{X X}$ lie on the right-hand side. However, these cases cannot be solved using our method, and therefore we do not consider them here.

It should also be noted that it is possible that the solutions obtained via this method will be susceptible to shear instability. This is a familiar shortcoming with inviscid hydraulics (for example Lawrence 1990 has shown that the two-layer exchange flow solution is invariably unstable) and must be borne in mind when applying internal hydraulic solutions to real flows. Finally, we highlight that the change of coordinates performed above can only be used for unidirectional flows. If there is a flow reversal within the domain - which will almost certainly happen in the case of a bidirectional exchange flow - then there must be two streamlines which pass through a given $X$-location with the same value. That is to say the $\eta$ coordinate must be multi-valued somewhere.

\subsection{Hydraulic equations}

In order to use the momentum equations (2.6) and (2.7) to solve for the evolution of velocity within the flow domain, we must be able to calculate the slope of the interface and free surface. This is done by extending the method of Hogg \& Hughes (2006). First, following Garrett \& Gerdes (2003), the continuity equation is used to express the lower-layer thickness in the new coordinate system

$$
h_{1} \equiv \int_{H}^{z_{I}} \mathrm{~d} z=\int_{0}^{\eta_{I}} z_{\eta} \mathrm{d} \eta=\int_{0}^{\eta_{I}} \frac{Q}{b u} \mathrm{~d} \eta .
$$


Equation (2.8) can then be differentiated to yield,

$$
\left(h_{1}\right)_{X}=-\int_{0}^{\eta_{I}} \frac{Q u_{X}}{b u^{2}} \mathrm{~d} \eta-\int_{0}^{\eta_{I}} \frac{Q b_{X}}{b^{2} u} \mathrm{~d} \eta .
$$

Substituting (2.6) into (2.9) and repeating the exercise for the second layer, yields two equations in layer height slopes. These can then be combined and rearranged (see Appendix A for details) to give

$$
\begin{aligned}
\left(h_{1}\right)_{X}\left(G^{2}-1\right)= & V_{1}\left((1-r) F_{1}^{2} F_{2}^{2}-F_{1}^{2}\right)+V_{2} r F_{2}^{2} \\
+ & \frac{b_{X}}{b}\left(r h_{2} F_{2}^{2}+(1-r) h_{1} F_{1}^{2} F_{2}^{2}-h_{1} F_{1}^{2}\right)+\left(1-F_{2}^{2}\right) H_{X}, \\
\left(h_{2}\right)_{X}\left(G^{2}-1\right)= & V_{1} F_{1}^{2}+V_{2}\left((1-r) F_{1}^{2} F_{2}^{2}-F_{2}^{2}\right) \\
& +\frac{b_{X}}{b}\left(h_{1} F_{1}^{2}+(1-r) h_{2} F_{1}^{2} F_{2}^{2}-h_{2} F_{2}^{2}\right)-F_{1}^{2} H_{X},
\end{aligned}
$$

where we have defined the Froude numbers,

$$
\begin{gathered}
\frac{1}{F_{1}^{2}} \equiv \int_{0}^{\eta_{I}} \frac{Q g^{\prime}}{b u^{3}} \mathrm{~d} \eta=\int_{H}^{z_{I}} \frac{g^{\prime}}{u^{2}} \mathrm{~d} z, \\
\frac{1}{F_{2}^{2}} \equiv \int_{\eta_{I}}^{\eta_{F}} \frac{Q g^{\prime}}{b u^{3}} \mathrm{~d} \eta=\int_{z_{I}}^{z_{F}} \frac{g^{\prime}}{u^{2}} \mathrm{~d} z .
\end{gathered}
$$

Here $g^{\prime}$ is the reduced gravity given by $g(1-r)$. The composite Froude number is in the same form as (1.1). We have also used a shorthand for the viscous terms,

$$
\begin{aligned}
V_{1} & \equiv \int_{0}^{\eta_{I}} \frac{v}{u^{2}}\left(\frac{u_{\eta}}{z_{\eta}}\right)_{\eta} \mathrm{d} \eta=\int_{H}^{z_{I}} \frac{v u_{z z}}{u^{2}} \mathrm{~d} z, \\
V_{2} & \equiv \int_{\eta_{I}}^{\eta_{F}} \frac{v}{u^{2}}\left(\frac{u_{\eta}}{z_{\eta}}\right)_{\eta} \mathrm{d} \eta=\int_{z_{I}}^{z_{F}} \frac{v u_{z z}}{u^{2}} \mathrm{~d} z .
\end{aligned}
$$

The Froude numbers defined in (2.12) and (2.13) are similar in form to those derived by Hogg \& Hughes (2006) and reduce to the usual definitions when viscosity is zero, and thus the velocity is constant within a layer. The equations in layer slopes, (2.10) and (2.11), are analogous to the hydraulic equations in two-layer inviscid flows (see e.g. Armi 1986). Hence we can define hydraulic control as occurring when the composite Froude number $G$ is unity, and by regularity of (2.11),

$$
V_{1} F_{1}^{2}+V_{2}\left((1-r) F_{1}^{2} F_{2}^{2}-F_{2}^{2}\right)=-\frac{b_{X}}{b}\left(h_{1} F_{1}^{2}+(1-r) h_{2} F_{1}^{2} F_{2}^{2}-h_{2} F_{2}^{2}\right)+\left(F_{1}^{2}\right) H_{X} .
$$

These two conditions are sufficient to ensure regularity of (2.10).

\subsection{Wave speeds at the control}

Hydraulic control is due physically to the arrest of wave modes at the critical point (e.g. Dalziel 1991). However, it is not clear from the above condition that the wave speeds are zero when $G^{2}$ is unity. Here we investigate the speed of inviscid interfacial waves in this flow and demonstrate that they are arrested at the hydraulic control.

Following Garrett \& Gerdes (2003) we linearize a time-dependent and inviscid version of (2.1) and (2.2) by separating the flow into a mean part $(U, W=0)$ and a 
wave component $\left(u^{\prime}, w^{\prime}\right)$. Within each layer we can write

$$
\begin{aligned}
& u_{t}^{\prime}+U u_{x}^{\prime}+w^{\prime} U_{z}=-g\left(h_{1 x}^{\prime}+r h_{2 x}^{\prime}\right), \quad 0<z<z_{I}, \\
& u_{t}^{\prime}+U u_{x}^{\prime}+w^{\prime} U_{z}=-g\left(h_{1 x}^{\prime}+h_{2 x}^{\prime}\right), \quad z_{I}<z<z_{F} .
\end{aligned}
$$

Assuming no bottom topography or width variation, the perturbation variables satisfy a continuity equation of the form

$$
u_{x}^{\prime}+w_{z}^{\prime}=0
$$

Now, consider a disturbance of sinusoidal form, i.e.

$$
\begin{aligned}
& u^{\prime}=u_{0}(z) \mathrm{e}^{\mathrm{i} k(x-c t)}, \\
& h_{i}^{\prime}=h_{i 0}(z) \mathrm{e}^{\mathrm{i} k(x-c t)} .
\end{aligned}
$$

Using (2.19) we can eliminate $u^{\prime}$ for $w^{\prime}$, giving

$$
\begin{aligned}
& (c-U) w_{z}^{\prime}+w^{\prime} U_{z}=-\mathrm{i} k g\left(h_{1}^{\prime}+r h_{2}^{\prime}\right), \quad 0<z<z_{I}, \\
& (c-U) w_{z}^{\prime}+w^{\prime} U_{z}=-\mathrm{i} k g\left(h_{1}^{\prime}+h_{2}^{\prime}\right), \quad z_{I}<z<z_{F} .
\end{aligned}
$$

By dividing by $(c-U)^{2}$, we can express the left-hand side of the above equations as exact derivatives. Then, integrating across the layers we have

$$
\begin{aligned}
& {\left[\frac{w^{\prime}}{c-U}\right]_{0}^{z_{I}}=-\mathrm{i} k g \int_{0}^{z_{I}} \frac{h_{1}^{\prime}+r h_{2}^{\prime}}{(c-U)^{2}} d z,} \\
& {\left[\frac{w^{\prime}}{c-U}\right]_{z_{I}}^{z_{F}}=-\mathrm{i} k g \int_{z_{I}}^{z_{F}} \frac{h_{1}^{\prime}+h_{2}^{\prime}}{(c-U)^{2}} d z .}
\end{aligned}
$$

The boundary conditions for these integrals are that $w=0$ at $z=0$, with kinematic conditions at the interface and free surface,

$$
\begin{array}{cc}
w^{\prime}=h_{1 t}^{\prime}+U h_{1 x}^{\prime}=-\mathrm{i} k(c-U) h_{1}^{\prime}, & z=z_{I}, \\
w^{\prime}=h_{1 t}^{\prime}+h_{2 t}^{\prime}+U h_{1 x}^{\prime}+U h_{2 x}^{\prime}=-\mathrm{i} k(c-U)\left(h_{1}^{\prime}+h_{2}^{\prime}\right), & z=z_{F} .
\end{array}
$$

This allows us to write the layer-integrated equations purely in terms of interface perturbation,

$$
\begin{aligned}
& -h_{1}^{\prime}=\int_{0}^{z_{I}} \frac{-g\left(h_{1}^{\prime}+r h_{2}^{\prime}\right)}{(c-U)^{2}} d z, \\
& -h_{2}^{\prime}=\int_{z_{I}}^{z_{F}} \frac{-g\left(h_{1}^{\prime}+h_{2}^{\prime}\right)}{(c-U)^{2}} d z .
\end{aligned}
$$

We can eliminate the interface heights from these equations to produce a single equation which is most simply written as

$$
\frac{g^{\prime}}{g}-\int_{0}^{z_{I}} \frac{g^{\prime}}{(c-U)^{2}} d z-\int_{z_{I}}^{z_{F}} \frac{g^{\prime}}{(c-U)^{2}} d z+\int_{0}^{z_{I}} \frac{g^{\prime}}{(c-U)^{2}} d z \int_{z_{I}}^{z_{F}} \frac{g^{\prime}}{(c-U)^{2}} d z=0 .
$$

The above equation can be compared with the critical condition when $c=0$, in which case

$$
(1-r)-F_{1}^{-2}-F_{2}^{-2}+F_{1}^{-2} F_{2}^{-2}=0,
$$

which is equivalent to $G^{2}=1$. In other words, when the critical condition is satisfied the internal wave speed is zero. Thus, we have demonstrated that the hydraulic control 
points defined above result in arrested internal waves, implying that the governing physics is identical to that of inviscid hydraulic theory.

\subsection{Numerical implementation}

We now outline the technique used to solve the hydraulic equations numerically. If, at a given position the velocity profile is specified, then (2.10) and (2.11) can be used to calculate the interfacial and free-surface slope. Furthermore, (2.5) can be used to express the momentum equations ((2.6) and (2.7)) in terms of the flux,

$$
\begin{array}{ll}
u_{X}=-\frac{g\left(H+h_{1}+r h_{2}\right)_{X}}{u}+\frac{b^{2} v}{2 Q^{3}}\left(Q\left(u^{2}\right)_{\eta \eta}-Q_{\eta}\left(u^{2}\right)_{\eta}\right), & 0<\eta<\eta_{I}, \\
u_{X}=-\frac{g\left(H+h_{1}+h_{2}\right)_{X}}{u}+\frac{b^{2} v}{2 Q^{3}}\left(Q\left(u^{2}\right)_{\eta \eta}-Q_{\eta}\left(u^{2}\right)_{\eta}\right), & \eta_{I}<\eta<\eta_{F} .
\end{array}
$$

These equations then allow downstream integration of the velocity along each streamline. This process breaks down when $G^{2}$ is close to unity, since the hydraulic equations are singular here. As in Hogg \& Hughes (2006), this can be circumvented by deriving an alternate equation for the layer slopes, valid in the vicinity of $G^{2}=1$. The alternate equation is obtained by finding the second derivative of the layer heights and combining them into a quadratic for $\left(H+h_{1}\right)_{X}$. The details of this derivation are given in Appendix B.

In the calculations that follow we set the velocity (flux) profile at the upstream end of the channel and integrate downstream until $G^{2}=1$. At this point the regularity requirement (2.16) must also be satisfied. This is achieved via a shooting algorithm in which the integration is iterated with varying upstream flux profiles until the control conditions are satisfied.

At this point it is advantageous to non-dimensionalize the governing equations in order to evaluate the results in a more general context. We scale the dimensional variables so that

$$
\begin{array}{r}
X \rightarrow \frac{X}{L}, \\
z \rightarrow \frac{z}{\eta_{0}}, \\
\eta \rightarrow \frac{\eta}{\eta_{0}}, \\
u \rightarrow \frac{u}{\sqrt{g^{\prime} \eta_{0}}}, \\
b \rightarrow \frac{b}{b_{0}},
\end{array}
$$

where $L$ is the characteristic length scale (half-width half-maximum) of the constriction or sill; $\eta_{0}$ is the height of the free surface at the upstream end; and $b_{0}$ is the upstream width of the channel. Using the non-dimensional variables the governing equations become

$$
\begin{aligned}
& u u_{X}=-\frac{\left(H+h_{1}+r h_{2}\right)_{X}}{1-r}+\frac{1}{\operatorname{ReA}} \frac{1}{z_{\eta}}\left(\frac{u_{\eta}}{z_{\eta}}\right)_{\eta}, \quad 0<\eta<\eta_{I}, \\
& u u_{X}=-\frac{\left(H+h_{1}+h_{2}\right)_{X}}{1-r}+\frac{1}{\operatorname{ReA}} \frac{1}{z_{\eta}}\left(\frac{u_{\eta}}{z_{\eta}}\right)_{\eta}, \quad \eta_{I}<\eta<\eta_{F} .
\end{aligned}
$$


(a) Plan view of constriction

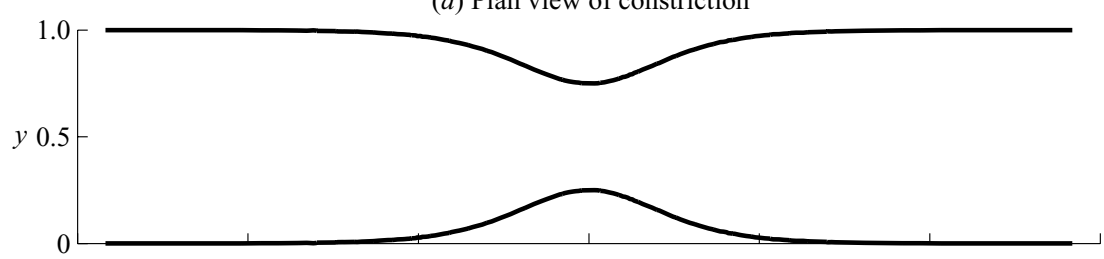

(b) Upper-layer-dominant downstream

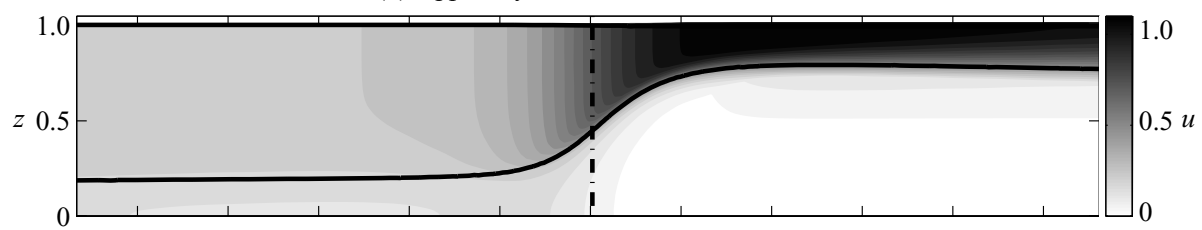

(c) Lower-layer-dominant downstream

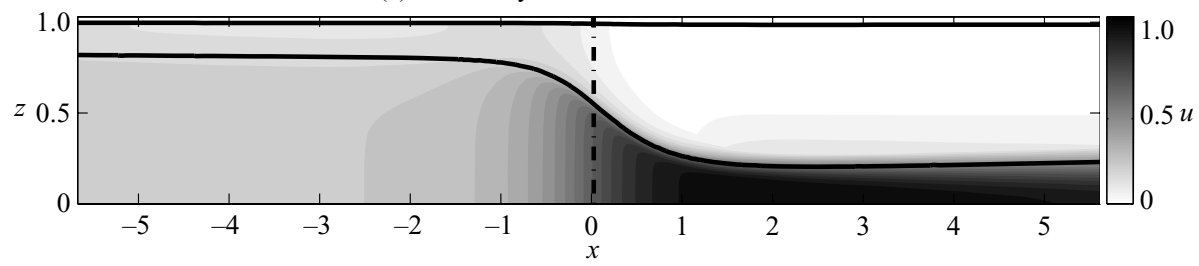

FIGURE 1. (a) Plan view of channel shape used in all simulations. The minimum width is half of that of the ends. $(b)$ Velocities and layer heights of the upper-layer-dominant solution with a viscosity of $v=3 \times 10^{-4}\left(1 /(\operatorname{Re} A)=7.9 \times 10^{-4}\right)$ and $\eta_{I}$ equal to 0.2 . The control point is approximately $0.024 L$ downstream of the constriction and is marked by the dash-dotted line. $(c)$ The lower-layer-dominant solution for the same viscosity as $(b)$ with $\eta_{I}=0.8$. The control point is slightly further downstream $(0.028 L)$, but otherwise the solution is a mirror image of $(b)$.

Here we have defined the Reynolds number $R e=\left(\sqrt{g^{\prime} \eta_{0}} \eta_{0}\right) / v$ and the channel aspect ratio $\eta_{0} / L$. This scaling is similar to that used in Hogg \& Hughes (2006), in which the velocity scale was taken as the speed of free-surface waves. Here, the relevant waveguide is the interface, and thus we use the speed of long interfacial waves in the absence of shear to non-dimensionalize the velocity. The Froude numbers and viscous terms take the dimensionless form,

$$
\begin{aligned}
\frac{1}{F_{1}^{2}} & =\int_{H}^{z_{I}} \frac{1}{u^{2}} d z \\
\frac{1}{F_{2}^{2}} & =\int_{z_{I}}^{z_{F}} \frac{1}{u^{2}} d z \\
V_{1} & =\int_{H}^{z_{I}} \frac{1}{\operatorname{ReA}} \frac{u_{z z}}{u^{2}} d z \\
V_{2} & =\int_{z_{I}}^{z_{F}} \frac{1}{\operatorname{ReA}} \frac{u_{z z}}{u^{2}} d z .
\end{aligned}
$$

We will use this scaling exclusively when describing the results in the next section.

Hydraulic solutions were calculated for flow through a lateral constriction of the form $b(x)=1-0.5 \cosh ^{-2}(x)$ (see figure $1 a$ ) with the density ratio $r \approx 0.99$ $\left(g^{\prime}=0.1 \mathrm{~m} \mathrm{~s}^{-2}\right)$. The calculations were performed for a channel which is of length $\approx 23 L$ and has a fine horizontal resolution $\left(4 \times 10^{4}\right.$ grid points $)$. This resolution was 
required in order to ensure accuracy of the first-order method used to integrate the equations downstream. A higher-order (RK4) method was tested but proved to be unstable.

At the upstream end the interface height $\eta_{I}$ was set depending on the type of controlled solution sought (see below). Standard simulations used 50 vertical layers, although sensitivity tests indicated that the solution was invariant provided more than 25 layers were used. The channel aspect ratio was set to $\eta_{0} / L=\ln (1+\sqrt{2}) \approx 0.88$ for all simulations, while viscosity was varied in the range $v=0-0.0012 \mathrm{~m}^{2} \mathrm{~s}^{-1}$, corresponding to Reynolds numbers of $1 /(\operatorname{Re} A)=0-0.0032$. The top and sidewall boundaries are free-slip in all cases. In order to concentrate on the effect of interfacial friction we initially consider the case in which the bottom boundary is also free-slip (§3.1-3.3), returning to the problem of bottom drag later (§3.4). The flux profile at the upstream end is given by

$$
Q(\eta)=\Omega\left(\tanh \left(\frac{\eta-\eta_{I}}{\delta}\right)+\beta\right),
$$

where $\delta$ is a measure of the thickness of the layer interface and was fixed at 0.0667 . Initially, $\beta$, the barotropic component, was fixed at 5.0, and $\Omega$ was varied to find the controlled solution. This process begins by integrating the hydraulic equations over the channel for a number of values of $\Omega$. Some of these solutions will be completely subcritical, while others will have points at which $G^{2}=1$. At these points the regularity condition (2.16) is evaluated. In the parameter region for which (2.16) is close to being satisfied a new set of solutions is derived, and the process is iterated until convergence is achieved. The parameter chosen to vary in this algorithm thus puts a constraint on the possible solutions that are allowed. In the case of varying $\Omega$ this means the ratio of flux between the upper and lower layers is the same in all solutions. In order to investigate the sensitivity of the results to the specifics of the solution process, simulations were also run with the absolute difference in the layer fluxes maintained (varying $\beta$ ) along with varying only the baroclinic component of the flow (varying $\Omega$ while holding $\Omega \beta$ constant).

\section{Results}

Two types of controlled solutions can be found, depending on which layer is faster at the upstream end of the channel. These two solutions are equivalent to the "upperlayer-dominant downstream' and 'lower-layer-dominant downstream' cases described by Armi \& Riemenschneider (2008). Representative solutions of each of these types are plotted in figure 1. In each case the upstream profiles are set so that the dominant layer is thicker and has a higher velocity at the upstream end. As the fluid approaches the control, the dominant layer accelerates and thins, with the highest velocities occurring between one and two half-widths downstream of the control. It is tempting to use the description of the dominant layer being 'controlled'; however this is not the case. The controlled solution requires knowledge of velocity in both layers, and thus both layers partake in setting control conditions.

Figures $1(b)$ and $1(c)$ show that the two different solutions are close to being mirror images of each other. This occurs because for a lateral constriction in which there is no bottom drag, the topographic effects are equal in each layer. The small amount of asymmetry that exists is due to a combination of non-Boussinesq effects (the free-surface deflection is of the order of $0.5 \%$ ) and a slight asymmetry in the upstream conditions (the dominant-layer thickness at the upstream end is $0.81 \eta_{0}$ in 
(a) Layer height and $\eta$-contours

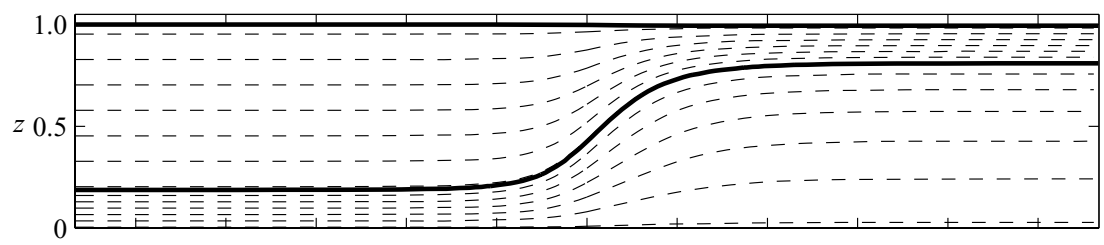

(b) Composite Froude number
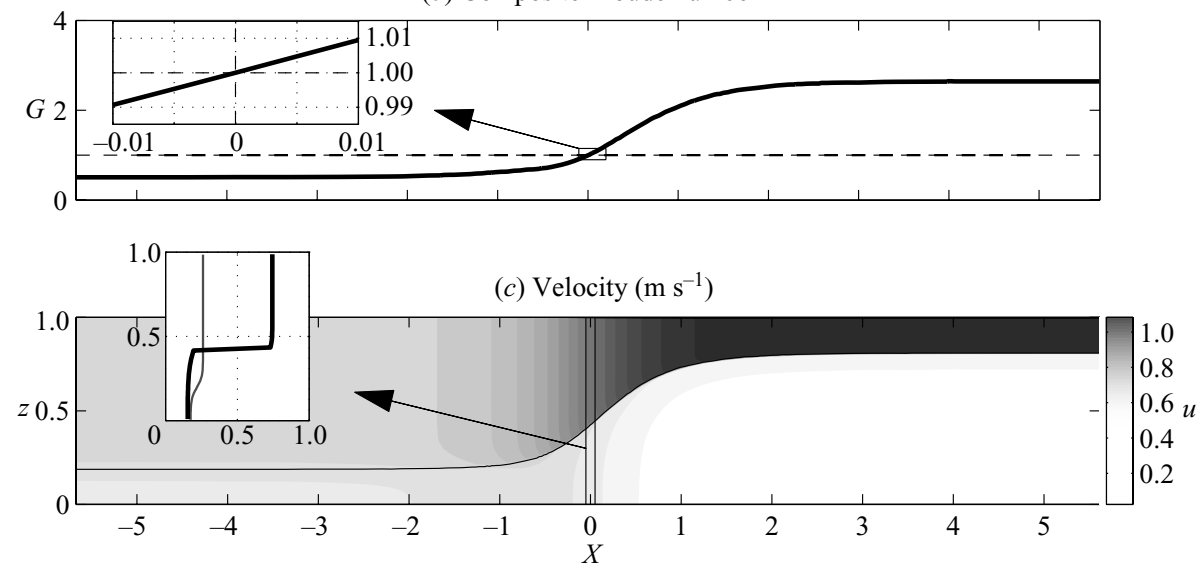

FIGURE 2. Upper-layer-dominant solution with no viscosity showing: (a) layer height and lines of constant $\eta$ showing the streamline-following coordinate system; $(b)$ the composite Froude number, with the inset showing the position of the control; $(c)$ velocity field, with the inset showing the velocity profile at the control point (thick line) and the upstream end (thin line).

figure $1 b$, while it is $0.82 \eta_{0}$ in figure $1 c$ ). Notwithstanding these small asymmetries, when considering solutions without bottom drag, we focus on results for the upperlayer-dominant solutions, with the implication that similar results exist for the lowerlayer-dominant case. In $\S 3.4$ we will consider the case in which there is friction with the lower boundary and will return to the differences in the upper- and lower-layerdominant solutions.

\subsection{Specific solutions}

Figure 2 shows the upper-layer-dominant solution for the inviscid case. The upstream height coordinate system (shown in figure $2 a$ ) is such that each layer has an equal number of streamlines to ensure that both layers are well resolved at the control. This requires that the dominant layer has a lower density of streamlines at the upstream end. The streamlines for the upper layer converge as the layer accelerates through the control, while in the lower layer flow decelerates with distance. The flow is controlled at the minimum width of the constriction (in keeping with the exact two-layer solution) and is supercritical downstream (figure $2 b$ ). Finally, the velocity profile at the control (figure $2 c$ ) shows a weak $z$-dependence within the layers, arising from the 'tanh' profile at the upstream boundary (shown in grey), while a sharp discontinuity in velocity develops at the interface. It is reasonable to expect that this discontinuity will be eroded when finite viscosity is used.

The viscous solution $\left(1 /(\operatorname{Re} A)=7.9 \times 10^{-4}\right)$ is shown in figure 3 . The primary features of interest in this case are the following: the velocity profile at the control point has been smoothed; the control is $0.024 \mathrm{~L}$ downstream of the topographic 
(a) Layer height and $\eta$-contours

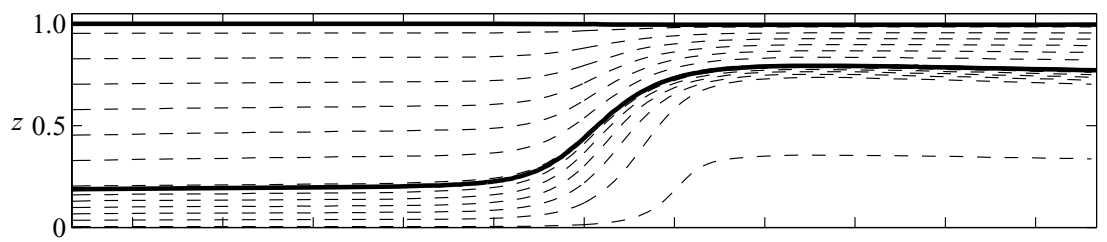

(b) Composite Froude number
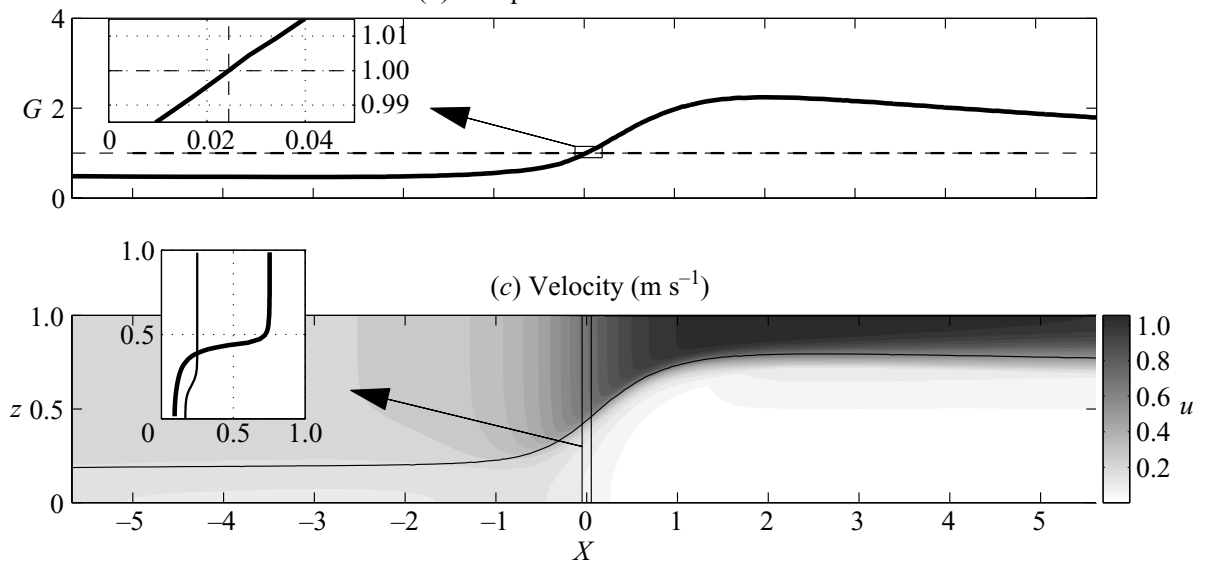

FIGURE 3. The same solution as in figure 2 , with a viscosity of $3 \times 10^{-4} \mathrm{~m}^{2} \mathrm{~s}^{-1}$ $\left(1 /(\operatorname{Re} A)=7.9 \times 10^{-4}\right)$.

minimum; and the velocity in the supercritical downstream region is reduced compared with that of the inviscid case. The smoothing of the velocity profile and loss of momentum downstream is an obvious consequence of viscosity; the behaviour of the control point is subtler and is tackled in detail in the following section.

The behaviour of the lower layer downstream of the control does not influence the physics at the control point but is nonetheless worth noting. There is a large region in the lower layer for which there are no streamlines, and almost zero velocity, that occurs in the viscous case but not in the inviscid case. This feature develops at relatively low viscosities $\left(1 /(\operatorname{ReA}) \approx 1.5 \times 10^{-4}\right)$ and is independent of vertical resolution. The implication of this result is that viscous flux of momentum from the upper to the lower layer, combined with the conservation of flux within a layer, works to generate a recirculation in the lower layer downstream of the control. The current formulation expressly forbids such a reversal. It is therefore likely that the solution in the downstream region is not accurate. However, as the integration is performed from the upstream end, as long as any such reversal remains downstream of the control, the dynamics at the control point are unchanged. On the other hand, the flow reversal may move into the control region at high viscosity, thus setting an upper bound on the viscosity range which can be used with this model.

\subsection{Control position}

In this section we examine the changes to the position of the hydraulic control with viscosity. Other parameters are the same as the solution in figures 2 and 3 . Figure 4(a) shows that the control position is monotonically shifted downstream from its position at the topographic minimum as viscosity is varied throughout the 


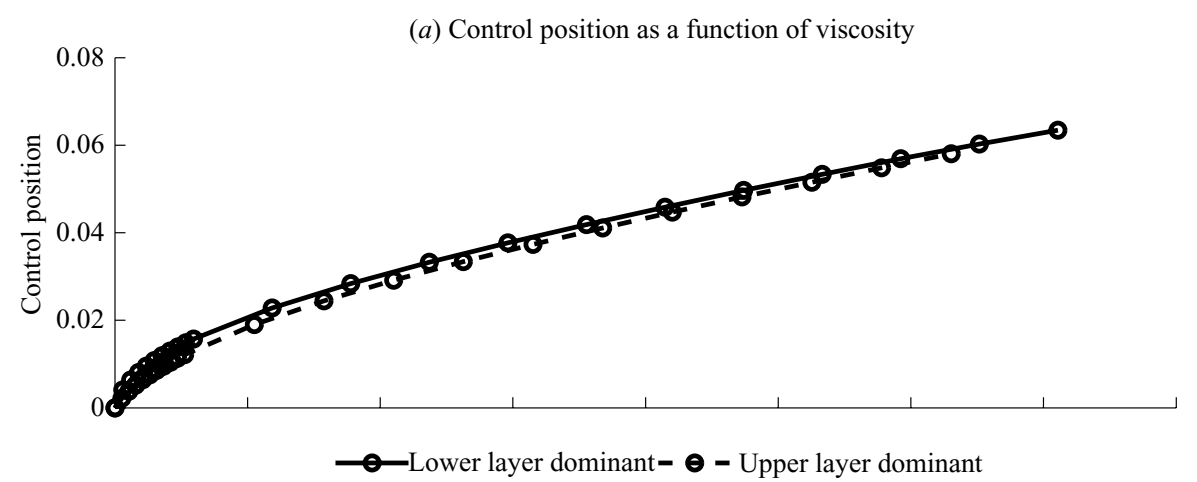

(b) Total flux for controlled solution

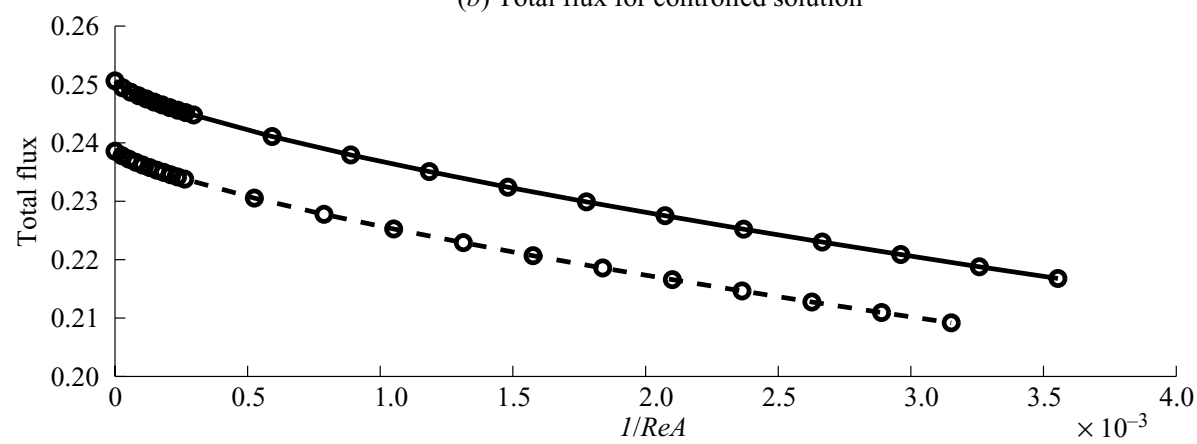

FIGURE 4. (a) Shift of the control point with increasing in viscosity. The control point moves monotonically downstream as viscosity is increased, as can be predicted from the hydraulic equations. (b) Decrease in flux for controlled solution as viscosity is increased.

range of viscosities which can be simulated with this technique. The downstream shift of the control point is modest - reaching a maximum of just $0.07 \mathrm{~L}$. However, a change in the control position can have global consequences in such flows. For example, the flux through the channel and the downstream layer depth are both sensitive to the control conditions.

The monotonic trend in control position with viscosity can be compared with results from one-layer flows studied in Hogg \& Hughes (2006), where the control point was found downstream of the topographic minimum, but the largest shift occurred for intermediate values of viscosity. They found that bottom drag moved the control downstream, while the weaker, internal friction moved it back upstream. Thus the magnitude of the shift depended upon the competing contributions of these two effects.

In the present case we have assumed free-slip boundaries, and hence there is no bottom drag, but there is friction at the interfacial boundary. The relative contributions of interfacial friction and viscosity within each layer can be examined by integrating (2.14) and (2.15) by parts to give

$$
\begin{aligned}
& V_{1}=\left[\frac{1}{\operatorname{ReA}} \frac{u_{z}}{u^{2}}\right]_{H}^{z_{I}}+\int_{H}^{z_{I}} \frac{2}{\operatorname{ReA} A} \frac{u_{z}^{2}}{u^{3}} d z=\frac{1}{\operatorname{ReA} A} \frac{u_{z}\left(z_{I}\right)}{u\left(z_{I}\right)^{2}}+\int_{H}^{z_{I}} \frac{2}{\operatorname{ReA} \frac{u_{z}^{2}}{u^{3}} d z} \\
& V_{2}=\left[\frac{1}{\operatorname{ReA}} \frac{u_{z}}{u^{2}}\right]_{z_{I}}^{z_{F}}+\int_{z_{I}}^{z_{F}} \frac{2}{\operatorname{ReA}} \frac{u_{z}^{2}}{u^{3}} d z=-\frac{1}{\operatorname{ReA}} \frac{u_{z}\left(z_{I}\right)}{u\left(z_{I}\right)^{2}}+\int_{z_{I}}^{z_{F}} \frac{2}{\operatorname{ReA}} \frac{u_{z}^{2}}{u^{3}} d z,
\end{aligned}
$$


where the lower- and upper-boundary terms are zero by the free-slip condition. Applying (3.1) and (3.2) to the regularity condition (2.16) and neglecting terms of order $(1-r)$ we can write

$$
\frac{u_{z}\left(z_{I}\right)}{u^{2}\left(z_{I}\right)}+F_{1}^{2}\left\{\int_{H}^{z_{I}} \frac{2 u_{z}^{2}}{u^{3}} d z\right\}-F_{2}^{2}\left\{\int_{z_{I}}^{z F} \frac{2 u_{z}^{2}}{u^{3}} d z\right\}=\operatorname{ReA} \frac{b_{X}}{b}\left(h_{2} F_{2}^{2}-h_{1} F_{1}^{2}\right),
$$

where the three terms on the left-hand side represent interfacial friction, and internal friction within layers 1 and 2 respectively. Given that $u_{z}\left(z_{I}\right)>0$ for the upper-layerdominant solutions, these three terms balance in such a way that interface friction and internal friction in the lower-layer shift the control downstream, while internal friction in the upper layer shifts it upstream. For the range of viscosities testable with this model, the interfacial friction term dominates. At the lowest viscosities this term is 10 times and 5 times the size of the layer 1 and layer 2 internal friction terms respectively. As the viscosity is increased, the relative contribution of layer 2 internal friction increases; however, this increase is not sufficient to move the control back upstream. Nevertheless, the competition between different physical processes is an important component of the solution, along with the possibility of upstream displacement of the control.

Equation (3.3) can be compared with the control condition that arises in drag models, where the velocity in each layer is considered to be constant with height. We consider the parameterization outlined in Zaremba et al. (2003). If only interface friction is considered, the viscous flux of momentum is taken as being proportional to the square of the velocity difference between the two layers. The control condition then becomes

$$
\frac{|\Delta u| \Delta u}{2 h_{1} h_{2}}=\frac{1}{f_{I} A} \frac{b_{X}}{b}\left(h_{2} F_{2}^{2}-h_{1} F_{1}^{2}\right),
$$

where $f_{I}$ is defined by the interfacial stress $\tau_{I}$, so that formally,

$$
f_{I}=\frac{2 \tau_{I}}{\rho|\Delta u| \Delta u} .
$$

That is to say the parameter $f_{I}$ is analogous to the Reynolds number in the present model. The major difference is that in the drag model prediction, there is no possibility of upstream displacement of the control, as the single term on the left-hand side of (3.2) will always move the control downstream. However, in the exchange flow solutions derived, Zaremba et al. (2003) found that the velocity difference $\Delta u$ decreases with increasing viscosity. This implies that at least for exchange flows, the movement of the control away from the topographic minimum is fastest at the lowest viscosities, qualitatively consistent with that found by our model.

The effect of viscosity on total flux through the channel is shown in figure 4(b). Flux decreases monotonically with increasing viscosity, as found for the case of one-layer flow in Hogg \& Hughes (2006). This is also consistent with drag model solutions found for exchange flows by Zaremba et al. (2003) and Gu \& Lawrence (2005). It should be noted, however, that this result pertains to cases in which the solution has been iterated through variation of the total amplitude of the profile (parameter $\Omega$ in 2.35). The selection of $\Omega$ as the parameter being varied constrains the solutions to maintain a constant flux ratio between the two layers. To eliminate the possibility of the variation in flux being dependent on the precise method used to find controlled solutions we also conducted experiments varying other parameters in (2.35). When $\beta$, the barotropic component of the flux, was varied, controlled solutions with the same 


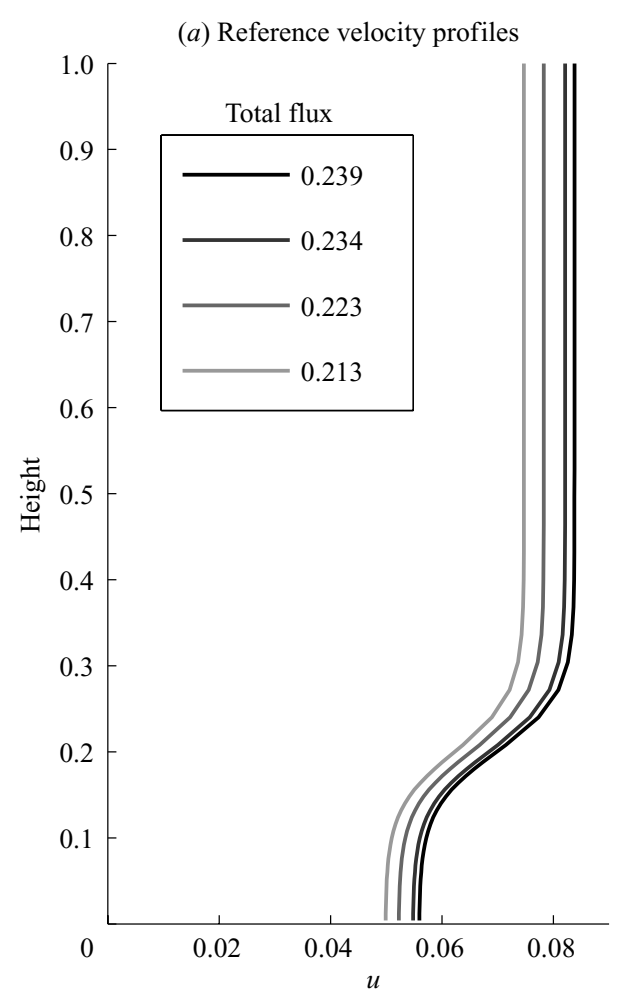

(b) Control velocity profiles

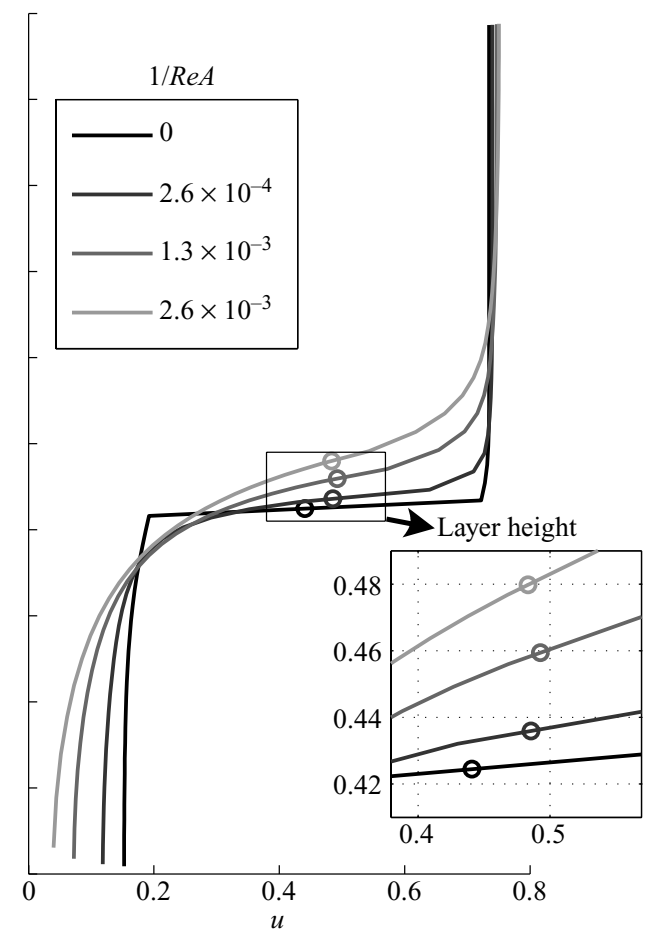

FIGURE 5. Vertical profiles of velocity $(a)$ at the upstream end and $(b)$ at the control for four different values of viscosity. The legends apply to both plots, with the darkest being the inviscid solution with the highest flux and the lightest being the solution with the highest viscosity and lowest flux. The inset in $(a)$ shows the height of the layer interface at the control for each solution.

dependence of flux upon viscosity were found. Varying the baroclinic component of velocity also produced a reduction in flux with viscosity, but the magnitude of that reduction was about half of that seen in figure $4(b)$. Thus we argue that the reduction in flux with increasing viscosity is a robust result and proceed to investigate the root cause of this behaviour by examining dynamics at the control point.

\subsection{Velocity distribution at the control point}

Figure 5 shows the velocity profile at $(a)$ the upstream end and $(b)$ the control point, for a number of upper-layer-dominant solutions with differing viscosities. The upstream profiles are simply the 'tanh' profiles given by $(2.35)$, with the parameter $\Omega$ adjusted so that the solution is controlled. At the control, the inviscid solution has the sharp discontinuity in velocity also shown in figure 2, which is gradually eroded with increasing viscosity. The reduction in velocity gradient is a consequence of the momentum transport from layer 2 to layer 1 . Surprisingly, however, the velocity in the lower part of layer 1 decreases with added viscosity, while the layer 2 velocity remains largely unchanged. Thus the top-to-bottom velocity difference is increased with viscosity.

If the first-order effect of viscosity is to transport momentum from the faster to the slower layer, it seems counter-intuitive that the top-to-bottom velocity difference increases. However, this result can be understood with reference to a simple rigid-lid two-layer case in which velocities are assumed to be constant with height. We compare 
two cases: one is inviscid and the other a viscous case in which a flux of momentum occurs across the interface dividing the two layers. The solution method of varying $\Omega$ in the upstream profile requires that the ratio of layer fluxes is unchanged between the two cases. That is to say

$$
Q_{1} / Q_{2}=Q_{1}^{\prime} / Q_{2}^{\prime}=1 / \alpha,
$$

where $Q_{i}=b h_{i} u_{i}$ is the layer flux in the inviscid case, and the primed variables are used to indicate the viscous case. For the upstream conditions used here, $\alpha=6$.

We assume that in the viscous case both velocity and layer height are altered so that

$$
\begin{array}{cc}
h_{1}^{\prime}=h_{1}+\delta h, & h_{2}^{\prime}=h_{2}-\delta h, \\
u_{1}^{\prime}=u_{1}+\delta u_{1}, & u_{2}^{\prime}=u_{2}+\delta u_{2},
\end{array}
$$

where the rigid-lid assumption implies that the changes in thickness in the two layers are equal and opposite.

Explicitly, we will write the layer fluxes in the viscous case as

$$
\begin{aligned}
& Q_{1}^{\prime} \approx Q_{1}+h_{1} \delta u_{1}+u_{1} \delta h, \\
& Q_{2}^{\prime} \approx Q_{2}+h_{2} \delta u_{2}-u_{2} \delta h,
\end{aligned}
$$

where we have linearized these equations about the inviscid case. Note that combining (3.4)-(3.6) gives

$$
h_{2} \delta u_{2}-u_{2} \delta h \approx \alpha h_{1} \delta u_{1}+\alpha u_{1} \delta h .
$$

In an uncontrolled flow, we expect that viscosity would act so as to reduce $u_{2}$ (i.e. $\delta u_{2}<0$ ) in exchange for increasing $u_{1}$ (i.e. $\delta u_{1}>0$ ) and for layer depths to adjust to conserve layer fluxes $(\delta h<0)$. However, when comparing two controlled flows, this does not hold because the controlled flow must satisfy

$$
F_{1}^{2}+F_{2}^{2} \approx 1
$$

at the topographic minimum. In the case we are considering, $F_{2}^{2} \gg F_{1}^{2}\left(F_{2}^{2} \approx 0.95\right)$ at the control, and so we simply write

$$
\frac{u_{2}^{\prime 2}}{h_{2}^{\prime}}=\frac{\left(u_{2}+\delta u_{2}\right)^{2}}{\left(h_{2}-\delta h\right)} \approx 1 .
$$

If the quadratic term is linearized and the control condition from the inviscid case removed, then

$$
\delta u_{2} \approx-\frac{\delta h}{2 u_{2}} .
$$

Thus, assuming that layer 2 loses momentum to layer $1,\left(\delta u_{2}<0\right)$, we find that $\delta h$ is positive. This represents a reduction in layer 2 thickness and a compensating increase in layer 1 thickness. This is confirmed by the inset in figure $5(b)$, showing the height of the interface at the control increasing with increasing viscosity.

Now that we have $\delta u_{2}$, we can also find $\delta u_{1}$ using (3.7) and assuming that $h_{2} \approx u_{2}^{2}$,

$$
\delta u_{1} \approx-\frac{\delta h}{h_{1}}\left(\frac{3 u_{2}}{2 \alpha}+u_{1}\right),
$$

showing that $\delta u_{1}$ is also negative. The relative magnitude of the velocity decrease of each layer is not clear from this approximate analysis, but the role of the control 


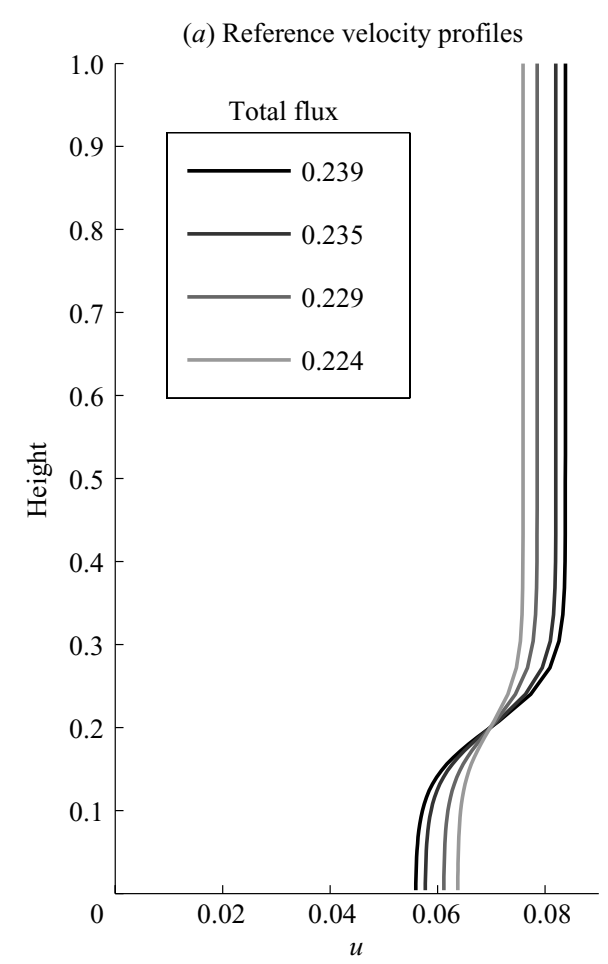

(b) Control velocity profiles

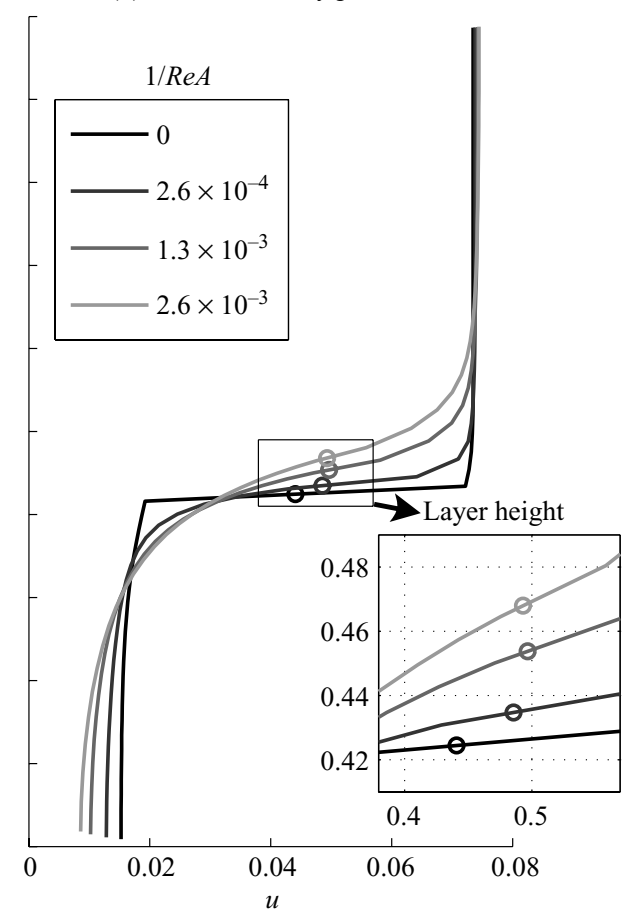

FIGURE 6. Same as figure 5 but varying the baroclinic component of the upstream velocity profile to find the controlled solution.

condition outlined here accounts for both layers decreasing in velocity in the calculated solutions.

The results shown above are robust to the upstream condition used. Figure 6 shows the same information as figure 5 but iterating over baroclinic velocity to find the controlled solution (i.e. varying $\Omega$ with the product $\Omega \beta$ held constant). This baroclinic variation can be seen clearly in the upstream profile (figure $6 a$ ), but the velocity profile at the control (figure $6 b$ ) is remarkably similar to the case shown in figure $5(b)$. This demonstrates that the controlled flow is insensitive to both degrees of freedom of the upstream profile, indicating that the governing dynamics in this flow is due to the constraints introduced by the hydraulic control.

\subsection{Effect of bottom drag}

In order to focus on the effects of interfacial friction on two-layer hydraulics we have, up until this point, neglected friction with the lower boundary. In this section we incorporate bottom drag using a formulation similar to that of Hogg \& Hughes (2006) in order to describe how this alters the solution.

As in Hogg \& Hughes (2006), friction with the lower boundary is incorporated into the solutions using a quadratic drag law so that (in dimensional variables)

$$
\left.v \frac{\partial \tilde{u}}{\partial \tilde{z}}\right|_{\tilde{z}=\tilde{H}}=\left.v \frac{\tilde{u}_{\tilde{\eta}}}{\tilde{z}_{\tilde{\eta}}}\right|_{\tilde{\eta}=0}=C_{d} \tilde{u}_{0}^{2},
$$

where $\tilde{u}_{0}$ is the velocity on the first $\tilde{\eta}$ contour and $C_{d}$ is a dimensionless drag coefficient. We have used tildes to emphasize that this equation is written in dimensional variables. 
Expressing the same equations non-dimensionally we have

$$
\left.\frac{1}{\operatorname{Re}} \frac{\partial u}{\partial z}\right|_{z=H}=\left.\frac{1}{\operatorname{Re}} \frac{u_{\eta}}{z_{\eta}}\right|_{\eta=0}=C_{d} u_{0}^{2} .
$$

This formulation parameterizes the subgrid-scale effects of a boundary layer on the bottom of the channel, without the need for a strict no-slip condition (which is not soluble in the streamline coordinate formulation). Controlled solutions for a number of bottom drag coefficients were calculated. As in Hogg \& Hughes (2006), an upper limit to the bottom drag coefficient existed, beyond which only subcritical solutions could be found. This occurred at a value of $C_{d}=0.04$. The solution process is identical to the free-slip lower boundary cases in that we use a 'tanh' profile at the upstream end as in (2.35) and vary the parameter $\Omega$ to find controlled solutions. Thus the ratio of fluxes between each layer remains constant in all simulations.

One major difference that would be expected between solutions with bottom drag compared with those previously described is the breaking of symmetry between the upper- and lower-layer-dominant solutions. Only the lower layer has an extra frictional term; thus the effects on the solution will be different depending on whether this is also the dominant layer. The numerical method used here produces smooth solutions for the upper-layer-dominant cases. However, the lower-layer-dominant solutions break down around the control when significant values of bottom drag are used. In these cases, poor convergence occurs because the alternate layer slopes equation derived for use as $G^{2}$ approaches unity (B 14) is not a sufficiently good approximation and causes non-smooth behaviour near the critical transition. We therefore show results for the lower-layer-dominant cases with small bottom drag that were able to be simulated accurately and otherwise focus the analysis on the upper-layer-dominant solution.

We begin by investigating the movement of the hydraulic control. With the above parameterization, the condition for a control in (3.3) can be written,

$\frac{u_{z}\left(z_{I}\right)}{u^{2}\left(z_{I}\right)}-F_{1}^{2} C_{d} R e+F_{1}^{2}\left\{\int_{H}^{z_{I}} \frac{2 u_{z}^{2}}{u^{3}} d z\right\}-F_{2}^{2}\left\{\int_{z_{I}}^{z_{F}} \frac{2 u_{z}^{2}}{u^{3}} d z\right\}=\operatorname{ReA} \frac{b_{X}}{b}\left(h_{2} F_{2}^{2}-h_{1} F_{1}^{2}\right)$.

Thus, for the upper-layer-dominant solution, $u_{z}\left(z_{I}\right)>0$, and bottom drag opposes interfacial friction, moving the control back upstream. However, in the lower-layerdominant case, the situation is reversed, and bottom drag pushes the control further downstream. The upper-layer-dominant case is demonstrated by figure $7(a)$, in which the variation of control position with viscosity is plotted for free-slip lower boundary and a bottom drag coefficient of $C d=0.04$ - the largest value for which controlled solutions were found. The shift of the control upstream by bottom drag is much smaller than the downstream shift by internal and interfacial friction. Much larger values of $C_{d}$ or, equivalently, much smaller values of the channel aspect ratio $A$ (for a given value of $1 / \operatorname{Re} A$ ) would be needed for bottom drag to significantly change the control position. Thus, in regard to control position at least, bottom friction is of secondary importance to viscosity within the fluid.

Figure $7(b)$ shows the variation of flux through the constriction under different viscosities and bottom drag coefficients, again for the upper-layer-dominant case only. Here the effect of bottom drag is more pronounced and in fact increases the flux compared with the free-slip case. This is a surprising result. Hogg \& Hughes (2006) found that bottom drag and internal friction decreased the flux in their one-layer 


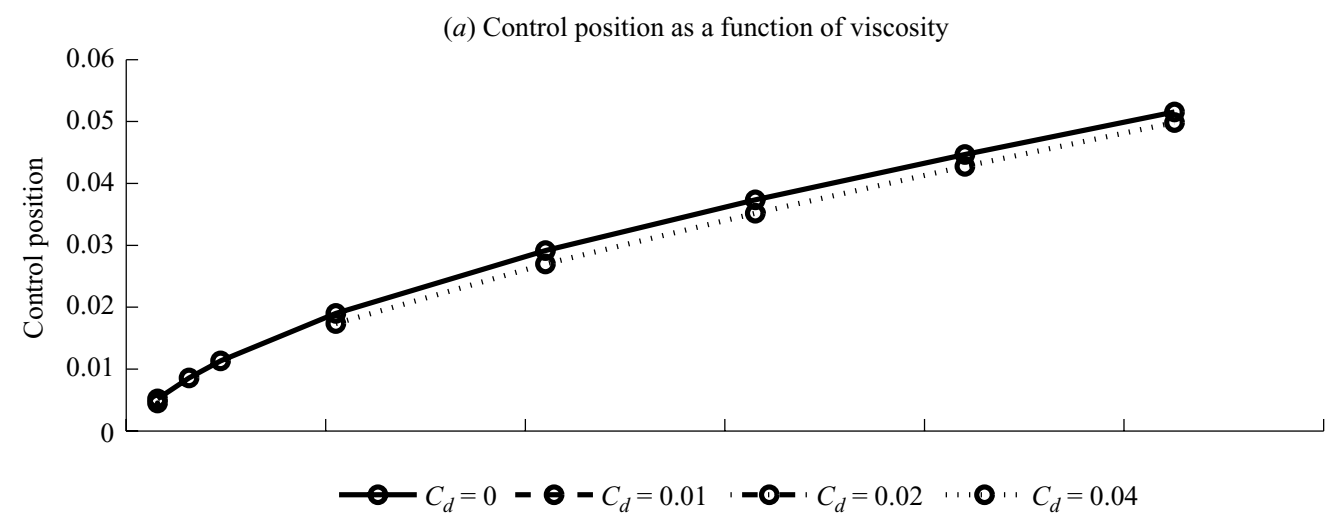

(b) Total flux for controlled solution

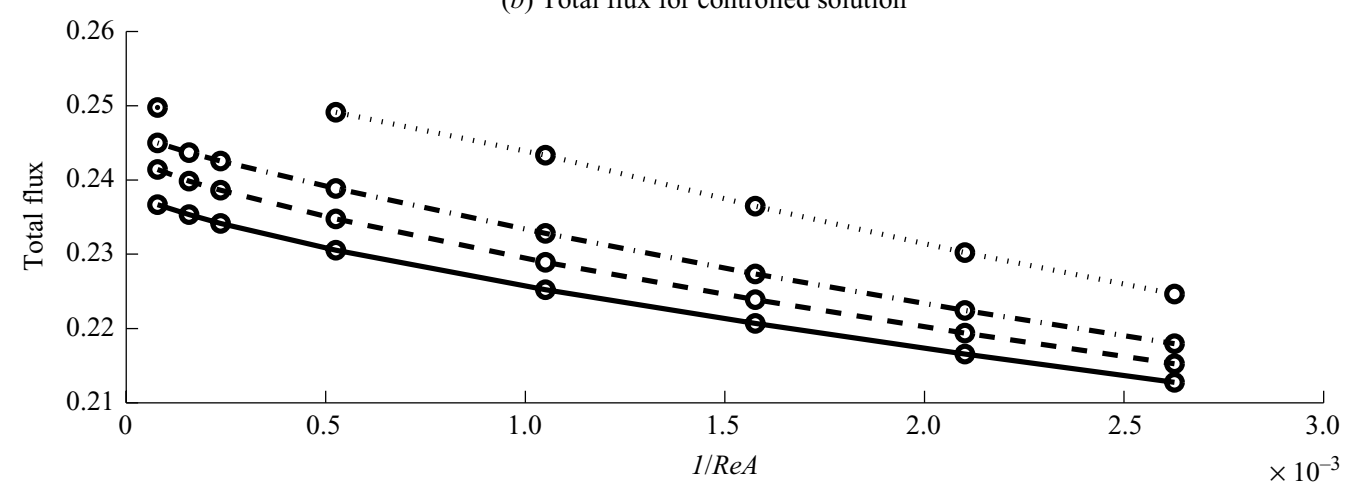

FIGURE 7. Upper-layer-dominant solution showing: (a) shift of the control point with increasing viscosity for various bottom drag coefficients and $(b)$ Flux through the constriction for various values of bottom drag and viscosity.

model, and similarly, using a drag model, Zaremba et al. (2003) found a decrease in the baroclinic flux in two-layer exchange flows. In this case, for certain combinations of bottom drag and internal viscosity, the flux in the controlled solution exceeds that of the inviscid solution. To investigate why this is so we again turn to the velocity profiles at the control point.

Figure 8 shows the velocity profiles at the control point for (a) low viscosity $\left(1 / \operatorname{Re} A=7.9 \times 10^{-5}\right)$ and $(b)$ high viscosity $\left(1 / \operatorname{Re} A=1.6 \times 10^{-3}\right)$ with varying bottom drag coefficient. The effect of bottom drag can clearly be seen in figure $8(a)$, with the lowest model levels being much more sluggish when bottom friction is turned on. In figure $8(b)$ the effects are smaller, and there is no obvious difference in the velocity of the fluid in the model levels closest to the boundary. The important point to note, however, is that in both cases the height of the interface at the control monotonically decreases with increasing bottom drag. We can show the connection between increasing flux decreasing interface height with the simple Boussinesq model outlined in $\S 3.3$.

Consider again (3.8) and (3.9) but this time linearized about the no-drag case. It can be seen that if the interface height decreases, both the upper- and lower-layer velocities increase, and thus the total flux increases. Conversely, if the interface height increases, the velocities and flux must decrease. Given that bottom drag takes momentum away from the lower layer, one might expect that the latter situation would occur. That the 
(a) $I / \operatorname{ReA}=7.9 \times 10^{-5}$

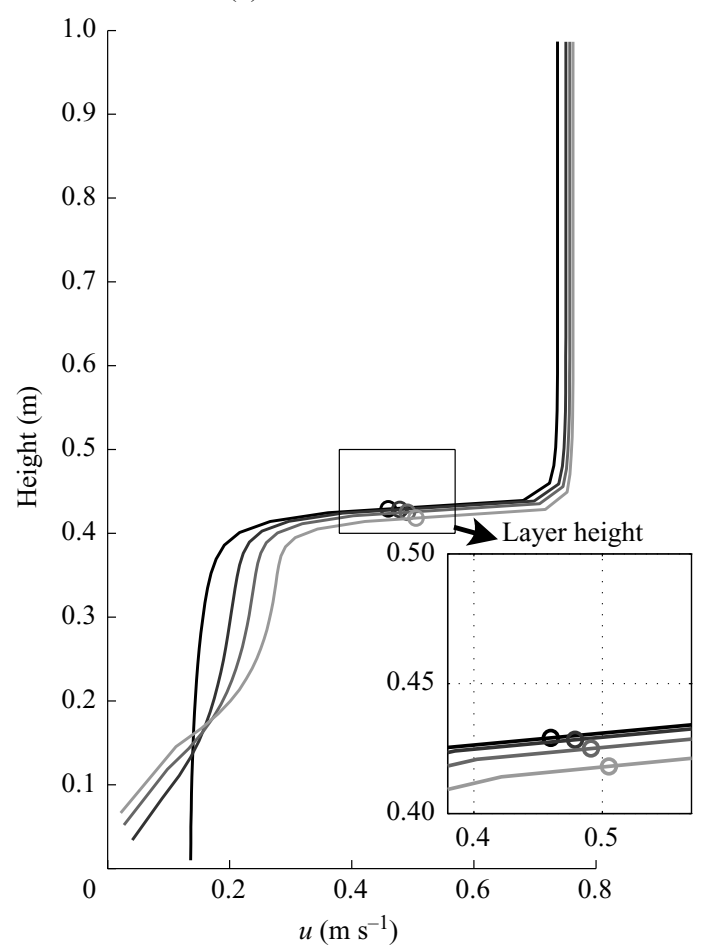

(b) $I / \operatorname{Re} A=1.6 \times 10^{-3}$

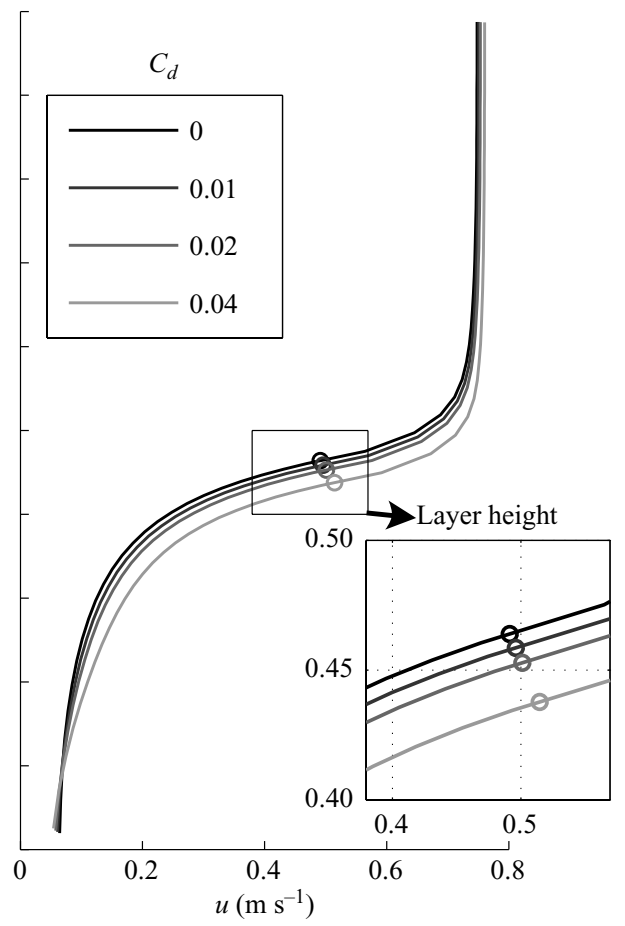

FIGURE 8 . Velocity profiles at the control, for varying bottom drag coefficient with viscosities such that $(a) 1 / \operatorname{Re} A=7.9 \times 10^{-5}$ and $(b) 1 / \operatorname{Re} A=1.6 \times 10^{-3}$.

reverse happens indicates that the sheared velocity profile interacts with the control condition to generate effects which cannot be predicted by simpler, layered hydraulic control conditions.

Figure 9 shows the effect of bottom drag on the lower-layer-dominant case. Because of the above-mentioned numerical difficulties, we have only shown solutions for the lowest value of bottom drag. Even these solutions had small discontinuities in the first derivative of layer height immediately downstream of the control. However, upstream of the control each of the solutions converged adequately; and as it is the control point which determines the flux, we argue that the results shown are accurate.

Figure $9(a)$ shows the position of the control with increasing viscosity. The control moved further downstream of the constriction with the inclusion of friction with the channel bottom. As for the upper-layer-dominant case, the movement was modest in comparison with the change because of internal viscosity. This result is well predicted by (3.12).

In figure $9(b)$, the total flux through the constriction is shown. Flux decreases as a result of friction with the lower boundary. This behaviour is opposite that of the upper-layer-dominant solutions but nevertheless consistent with the arguments compiled in $\S 3.3$. When bottom drag is added, the dominant (lower) layer now loses momentum to the boundary and is thinner at the control (not shown). When (3.8) and (3.9) are written for the lower-layer-dominant case, this leads to a decrease in the velocity of both layers, and hence a decrease in the flux. 

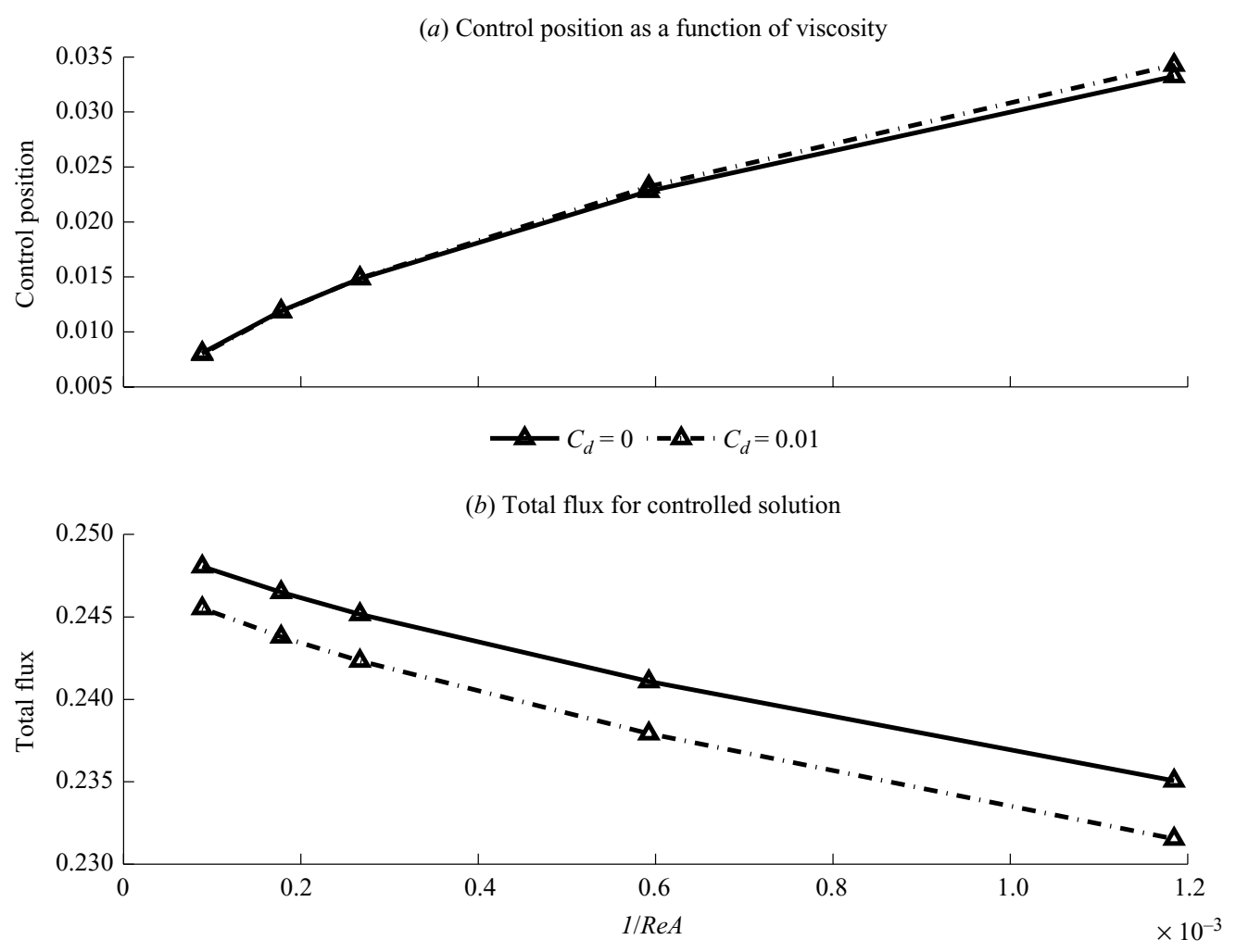

FIGURE 9. Same as figure 7 but for the lower-layer-dominant case.

\section{Discussion and conclusions}

The solution presented in this paper is a new method of solving for hydraulic control of vertically sheared, but immiscible, layers of differing density. The technique has a number of restrictions - most notably that the streamline-following coordinate system cannot handle reversals in the flow as it becomes multi-valued. This means that bidirectional flow cannot be addressed with this technique and that the range of viscosities which can be modelled is limited by the occurrence of flow reversals in the slowest layer. In addition, the solutions prohibit mixing between the layers, by only considering constant-density layers. However, the solution does provide considerable insight into the problem of layered hydraulic control.

The first insight is that we can define Froude numbers, according to (2.12) and (2.13), which involve integrating the inverse velocity squared. These definitions revert to the layered Froude numbers when velocities are constant but give a different result from the standard method of averaging layer velocities to estimate the Froude numbers. The physical explanation for this is that shear plays a role in modifying internal wave speeds, and thus the Froude number (the effective ratio of flow speed to wave speed) is increased when shear is amplified. These Froude numbers are consistent with recent results of Pratt (2008) which pertain to integrating the inverse square of velocity across a fluid layer to measure hydraulic control in a fluid with horizontal shear.

We have shown that the new Froude numbers, remarkably, result in the same relationship between the layer and the composite Froude numbers, that the composite 
Froude number critical condition remains $G^{2}=1$ and that the critical condition equates to the arrest of inviscid internal waves. This means that the solutions presented here are consistent with the inviscid results for unidirectional flow presented by Armi (1986). In addition, it provides encouragement that in future work, Froude numbers incorporating shear in the velocity profile may be incorporated into multi-layer solutions. However, there is a contradiction between this result and the inference made by Hogg, Winters \& Ivey (2001b) who solved a sixth-order viscous-diffusive wave equation to estimate criticality. They found broad consistency between the position of control points and those estimated by the wave equation, implying that the viscosity and diffusion play a role in setting control points. The present results refute that implication: here we derive an exact control condition (albeit for an ideal non-diffusive case) and find that the role of viscosity is in creating shear, rather than directly influencing the control criteria. There is no reason to expect that the addition of diffusion will alter this result, but this cannot yet be ruled out.

The new Froude numbers may make it possible to more accurately gauge the criticality or otherwise of observed flow measured in the mouths of estuaries or in ocean straits. Here the strategy would be to divide the flow into defined constantdensity layers and use the velocity shear within each layer to refine estimates of the layer Froude numbers. Such a process could take into account the effect of shear in governing the Froude number but not continuous stratification. However, the definition of the layer Froude numbers involves the integral of the inverse square of velocity, so that if velocity approaches zero at any location, the singularities in the equation cause the layer Froude number to approach zero. This result pertains physically to the effect of a sluggish layer acting to decouple two faster-flowing regions (Engqvist 1996; Hogg \& Killworth 2004; Pratt 2008). In trying to apply this result to a continuously stratified fluid there is a clear sensitivity on the definition of layers, which may make such a technique practically impossible. Investigation of the application of this technique is left for future studies.

The second insight we gain from the present approach is the counter-intuitive result that the top-to-bottom velocity difference is enhanced by the introduction of viscosity. This result can be understood by assuming that upper-layer velocity decreases because of loss of momentum to the lower layer. The control condition requires a reduction in upper-layer thickness, and this results in a decrease in lower-layer velocity to ensure conservation of mass within each layer. This illustrates the way that hydraulics controls the global solution, by specification of the conditions at just one point.

The addition of internal friction pushes the control point downstream, primarily because interfacial friction increases the curvature of the velocity profile, thereby altering the regularity requirements on the topographic control. Internal viscosity within the lower layer enhances the effect of interfacial friction, while internal viscosity in the upper layer acts to shift the control point back upstream. The possibility of a downstream reversal of the flow direction with increasing shear is demonstrated.

The introduction of a frictional lower boundary also produces some new and interesting results. While bottom friction has only a small effect on the control position (shifting it upstream) the effect on the flux through the constriction is marked. Most surprisingly, for upper-layer-dominant solutions, bottom drag increases the flux through the channel, in some cases to a value greater than that of the inviscid solution. This is contrary to internal friction which monotonically reduces the flux in 
both upper- and lower-dominant cases. Previous results for one-layer flows (Hogg \& Hughes 2006) and drag model solutions of two-layer exchange flows (Zaremba et al. 2003) all found that friction (both internal and with the boundaries) reduced the flux in hydraulically controlled flows. These results demonstrate the important role that friction may play in hydraulically controlled flows and the insight that can be gained by retaining a continuous velocity profile that may not be possible with the classic drag model approach.

This work was conducted while M.S.S. was at ANU on a Summer Research Scholarship. The late Peter Killworth provided valuable advice on the use of streamfunction coordinates during the early stages of this project. Suggestions from Larry Armi and Graham Hughes helped to substantially improve the first draft of this manuscript. Two anonymous reviewers provided a number of constructive comments on an earlier submission of this manuscript.

\section{Appendix A. Derivation of hydraulic equations}

Here we provide the details of the derivation of the hydraulic equations (2.10) and (2.11). Substituting for $u_{X}$ in (2.9) and the corresponding equation for $\left(h_{2}\right)_{X}$ we have

$$
\begin{aligned}
& \left(h_{1}\right)_{X}=\frac{\left(H+h_{1}+r h_{2}\right)_{X}}{(1-r) F_{1}^{2}}-V_{1}-\frac{h_{1} b_{X}}{b}, \\
& \left(h_{2}\right)_{X}=\frac{\left(H+h_{1}+h_{2}\right)_{X}}{(1-r) F_{2}^{2}}-V_{2}-\frac{h_{2} b_{X}}{b},
\end{aligned}
$$

where we have expressed all quantities in terms of the definitions in (2.12)-(2.15). Eliminating $\left(h_{2}\right)_{X}$ we have

$$
\begin{aligned}
\left(h_{1}\right)_{X}\left(1-\frac{1}{(1-r) F_{1}^{2}}-\frac{1}{(1-r) F_{2}^{2}}+\frac{1}{(1-r) F_{1}^{2} F_{1}^{2}}\right) \\
=-\left(V_{1}+\frac{h_{1} b_{X}}{b}\right)\left(1-\frac{1}{(1-r) F_{2}^{2}}\right)-\left(V_{2}+\frac{h_{2} b_{X}}{b}\right) \frac{r}{(1-r) F_{1}^{2}} \\
\quad+H_{X}\left(\frac{1}{(1-r) F_{1}^{2}}-\frac{1}{(1-r) F_{1}^{2} F_{2}^{2}}\right)
\end{aligned}
$$

Multiplying through by $F_{1}^{2} F_{2}^{2}(1-r)$ gives

$$
\begin{aligned}
\left(h_{1}\right)_{X} & \left((1-r) F_{1}^{2} F_{2}^{2}-F_{2}^{2}-F_{1}^{2}+1\right) \\
= & -\frac{b_{X}}{b}\left((1-r) h_{1} F_{1}^{2} F_{2}^{2}-h_{1} F_{1}^{2}+r h_{2} F_{2}^{2}\right) \\
& \quad-V_{1}\left((1-r) F_{1}^{2} F_{2}^{2}-F_{1}^{2}\right)-V_{2} r F_{2}^{2}+H_{X}\left(F_{2}^{2}-1\right),
\end{aligned}
$$

which with a little rearrangement is (2.10). Equation (2.11) is obtained by substituting (A 4) into (A 2). 


\section{Appendix B. Derivation of alternate layer slope equation}

In this section we derive the quadratic used to find the layer slopes when $G^{2} \approx 1$. Differentiating (A 1) and (A 2) we have

$$
\begin{aligned}
\left(h_{1}\right)_{X X}= & \frac{1}{1-r}\left(\frac{\left(H+h_{1}+r h_{2}\right)_{X X}}{F_{1}^{2}}+\left(H+h_{1}+r h_{2}\right)_{X}\left(\frac{1}{F_{1}^{2}}\right)_{X}\right) \\
& -\left(V_{1}\right)_{X}-\frac{h_{1} b_{X} X}{b}-\frac{\left(h_{1}\right)_{X} b_{X}}{b}+\frac{h_{1} b_{X}^{2}}{b^{2}} \\
\left(h_{2}\right)_{X X}= & \frac{1}{1-r}\left(\frac{\left(H+h_{1}+h_{2}\right)_{X X}}{F_{2}^{2}}+\left(H+h_{1}+h_{2}\right)_{X}\left(\frac{1}{F_{2}^{2}}\right)_{X}\right) \\
& -\left(V_{2}\right)_{X}-\frac{h_{2} b_{X} X}{b}-\frac{\left(h_{2}\right)_{X} b_{X}}{b}+\frac{h_{2} b_{X}^{2}}{b^{2}} .
\end{aligned}
$$

Eliminating $\left(h_{2}\right)_{X X}$ and multiplying through by $(1-r) F_{1}^{2} F_{2}^{2}$ gives

$$
\begin{aligned}
\left(h_{1}\right)_{X X}\left(1-G^{2}\right)= & r F_{2}^{2} \frac{\left(H+h_{1}+h_{2}\right)_{X}}{(1-r)}\left(\frac{1}{F_{2}^{2}}\right)_{X} \\
& +\left((1-r) F_{1}^{2} F_{2}^{2}-F_{1}^{2}\right) \frac{\left(H+h_{1}+r h_{2}\right)_{X}}{(1-r)}\left(\frac{1}{F_{1}^{2}}\right)_{X} \\
& +r F_{2}^{2}\left(\frac{h_{2} b_{X}^{2}}{b^{2}}-\frac{h_{2} b_{X} X}{b}-\frac{\left(h_{2}\right)_{X} b_{X}}{b}\right) \\
& +\left((1-r) F_{1}^{2} F_{2}^{2}-F_{1}^{2}\right)\left(\frac{h_{1} b_{X}^{2}}{b^{2}}-\frac{h_{1} b_{X} X}{b}-\frac{\left(h_{1}\right)_{X} b_{X}}{b}\right) \\
& +(H)_{X X}\left(F_{2}^{2}-1\right)-\left((1-r) F_{1}^{2} F_{2}^{2}-F_{1}^{2}\right)\left(V_{1}\right)_{X}-r F_{2}^{2}\left(V_{2}\right)_{X} .
\end{aligned}
$$

Now, differentiating (2.12)-(2.15) we have

$$
\begin{aligned}
\frac{1}{(1-r)}\left(\frac{1}{F_{1}^{2}}\right)_{X} & =I_{1}^{5}\left(H+h_{1}+h_{2}\right)_{X}-\frac{3}{2} I_{2}^{4}-\frac{b_{X}}{b(1-r) F_{2}^{2}}, \\
\frac{1}{(1-r)}\left(\frac{1}{F_{2}^{2}}\right)_{X} & =I_{2}^{5}\left(H+h_{1}+r h_{2}\right)_{X}-\frac{3}{2} I_{1}^{4}-\frac{b_{X}}{b(1-r) F_{1}^{2}}, \\
\left(V_{1}\right)_{X} & =I_{1}^{4}\left(H+h_{1}+r h_{2}\right)_{X}-I_{1}^{3}+I_{1}^{2}+E_{1}, \\
\left(V_{2}\right)_{X} & =I_{2}^{4}\left(H+h_{1}+h_{2}\right)_{X}-I_{2}^{3}+I_{2}^{2}+E_{2},
\end{aligned}
$$

where, for brevity, we have defined the integrals

$$
\begin{aligned}
I_{i}^{2} & =\int \frac{b_{X} v}{2 Q^{2} u^{2}}\left(Q\left(u^{2}\right)_{\eta \eta}-Q_{\eta}\left(u^{2}\right)_{\eta}\right) d \eta, \\
I_{i}^{3} & =\int \frac{b^{3} v^{2}}{2 Q^{5} u^{3}}\left(Q\left(u^{2}\right)_{\eta \eta}-Q_{\eta}\left(u^{2}\right)_{\eta}\right) d \eta, \\
I_{i}^{4} & =\int \frac{g b v}{Q^{2} u^{4}}\left(Q\left(u^{2}\right)_{\eta \eta}-Q_{\eta}\left(u^{2}\right)_{\eta}\right) d \eta, \\
I_{i}^{5} & =\int \frac{3 Q g^{2}}{b u^{5}}\left(Q\left(u^{2}\right)_{\eta \eta}-Q_{\eta}\left(u^{2}\right)_{\eta}\right) d \eta, \\
E_{i} & =\int \frac{b v}{2 Q^{2} u^{2}}\left(Q\left(u^{2}\right)_{\eta \eta X}-Q_{\eta}\left(u^{2}\right)_{\eta X}\right) d \eta .
\end{aligned}
$$


Here the subscript $i$ refers to the layer over which the integration is performed. Finally, we can write (A 1) as

$$
\left(H+h_{1}+r h_{2}\right)_{X}=(1-r) F_{1}^{2}\left(\left(h_{1}\right)_{X}-V_{1}-\frac{h_{1} b_{X}}{b}\right) .
$$

Assuming $G^{2} \approx 1$ we can combine (B 3)-(B 13) and write a quadratic in $\left(H+h_{1}\right)_{X}$,

$$
\begin{aligned}
& 0=\left\{\left[\left(F_{2}^{2}-G^{2}\right) I_{1}^{5}+\left(1-\frac{1}{F_{2}^{2}}\right)^{2} \frac{F_{2}^{2}}{r} I_{2}^{5}\right](1-r)^{2} F_{1}^{4}\right\}\left(H+h_{1}\right)_{X}^{2}, \\
& +\left\{\begin{array}{c}
-\frac{5(1-r) F_{1}^{2} I_{1}^{4}}{2}\left(F_{2}^{2}-G^{2}\right)+\frac{5(1-r) F_{2}^{2} I_{2}^{4}}{2}\left(1-F_{1}^{2}\right) \\
+\frac{b_{X}}{b}\left(\left(G^{2}+1-2 F_{1}^{2} F_{2}^{2}(1-r)\right)\right. \\
+2(1-r)^{2} F_{1}^{4}\left(V_{1}+\frac{h_{1} b_{x}}{b}\right)\left(\left(F_{2}^{2}-G^{2}\right) I_{1}^{5}+\frac{F_{2}^{2}}{r} I_{2}^{5}\left(1-\frac{1}{F_{1}^{2}}\right)\right)
\end{array}\right\}\left(H+h_{1}\right)_{X} \\
& +\left((1-r) F_{1}^{2} F_{2}^{2}-F_{1}^{2}\right)\left(\frac{H_{X} b_{X}}{b}\right)+\left(F_{2}^{2}-1\right) H_{X X} \\
& +\left(F_{2}^{2}-G^{2}\right)\left(I_{1}^{3}-I_{1}^{2}\right)+r F_{2}^{2}\left(I_{2}^{3}-I_{2}^{2}\right) \\
& +\left(\left(F_{2}^{2}-G^{2}\right) h_{1}+r F_{2}^{2} h_{2}\right)\left(\frac{b_{X}^{2}}{b^{2}}-\frac{b_{X X}}{b}\right) \\
& +\left(\left(F_{2}^{2}-G^{2}\right) I_{1}^{5}+\frac{F_{2}^{2}}{r} I_{2}^{5}\right)(1-r)^{2} F_{1}^{4}\left(V_{1}+\frac{h_{1} b_{X}}{b}\right)^{2} \\
& -\left[\left(F^{2}-G^{2}\right) \frac{5(1-r) F_{1}^{2} I_{1}^{4}}{2}+\frac{5(1-r) F_{2}^{2} I_{2}^{4}}{2} F_{1}^{2}+\frac{b_{X}}{b}\left(2 F_{1}^{2} F_{2}^{2}(1-r)\right)\right]\left(V_{1}+\frac{h_{1} b_{X}}{b}\right) \\
& -\left(F_{2}^{2}-G^{2}\right) E_{1}-r F^{2} E_{2} .
\end{aligned}
$$

This equation can be solved if it is assumed that the terms involving the $X$-gradient of the shear $\left(E_{1}\right.$ and $\left.E_{2}\right)$ are small enough to be neglected. This assumption is confirmed a posteriori.

\section{REFERENCES}

Armi, L. 1986 The hydraulics of two flowing layers with different densities. J. Fluid Mech. 163, $27-58$.

ARMi, L. \& FARMER, D. M. 1986 Maximal two-layer exchange through a contraction with barotropic net flow. J. Fluid Mech. 164, 27-51.

Armi, L. \& Riemenschneider, U. 2008 Two-layer hydraulics for a co-located crest and narrows. $J$. Fluid Mech. 615, 169-184.

Dalziel, S. B. 1991 Two layer hydraulics: a functional approach. J. Fluid Mech. 223, 135-163.

DAlziel, S. B. 1992 Maximal exchange in channels with nonrectangular cross-sections. J. Phys. Oceanogr. 22, 1188-1206.

ENGQvist, A. 1996 Self-similar multi-layer exchange flow through a contraction. J. Fluid Mech. 328, 49-66.

Garrett, C. 2004 Frictional processes in straits. Deep-Sea Res. II 51, 393-410.

Garrett, C. \& Gerdes, F. 2003 Hydraulic control of homogeneous shear flows. J. Fluid Mech. 475, $163-172$.

Gu, L. \& Lawrence, G. A. 2005 Analytical solution for maximal frictional two-layer exchange flow. J. Fluid Mech. 543, 1-17. 
Hogg, A. M. \& Hughes, G. O. 2006 Shear flow and viscosity in single-layer hydraulics. J. Fluid Mech. 548, 431-443.

Hogg, A. M., Ivey, G. N. \& Winters, K. B. 2001a Hydraulics and mixing in controlled exchange flows. J. Geophys. Res. 106, 959-972.

HogG, A. M. \& Killworth, P. D. 2004 Continuously stratified exchange flow through a contraction in a channel. J. Fluid Mech. 499, 257-276.

Hogg, A. M., Winters, K. B. \& Ivey, G. N. $2001 b$ Linear internal waves and the control of stratified exchange flows. J. Fluid Mech. 447, 357-375.

Lawrence, G. A. 1990 On the hydraulics of Boussinesq and non-Boussinesq two-layer flows. $J$. Fluid Mech. 215, 457-480.

PratT, L. J. 2008 Critical conditions and composite Froude numbers for layered flow with transverse variations in velocity. J. Fluid Mech. 605, 281-291.

Wood, I. R. 1968 Selective withdrawal from a stably stratified fluid. J. Fluid Mech. 32, 209-223.

Zaremba, L. J., Lawrence, G. A. \& Pieters, R. 2003 Frictional two-layer exchange flow. J. Fluid Mech. 474, 339-354. 